\section{BMJ Paediatrics Open}

\title{
Audit of child maltreatment medical assessments in a culturally diverse, metropolitan setting
}

\author{
Shanti Raman, ${ }^{1}$ Paul Rex Hotton ${ }^{2}$
}

To cite: Raman S, Hotton PR. Audit of child maltreatment medical assessments in a culturally diverse, metropolitan setting. BMJ Paediatrics Open 2017;1:e000125. doi:10.1136/ bmjpo-2017-000125

Received 31 May 2017 Accepted 7 November 2017

\section{CrossMark}

${ }^{1}$ Department of Community Paediatrics, South Western Sydney Local Health District, Liverpool, New South Wales, Australia

${ }^{2}$ Community Child Health, Sydney Children's Hospital, Randwick, New South Wales, Australia

Correspondence to Dr Shanti Raman; Shanti. Raman@sswahs.nsw.gov.au

\section{ABSTRACT}

Objective Child maltreatment (CM) is a major public health problem globally. While there is evidence for the value of medical examination in the assessment of $\mathrm{CM}$, little is known about the quality of clinical assessments for CM. South Western Sydney (SWS) has a large metropolitan population with many vulnerable subgroups. We aimed to describe acute presentations of CM in SWS over a 3 -year period - with a focus on the quality of the clinical assessments. We wanted to determine whether the cases assessed fulfilled established minimum standards for clinical assessment of $\mathrm{CM}$ and whether the assessments were performed in a child-friendly manner.

Design We gathered data from the acute child protection database on all children $<16$ years referred for assessment between 2013 and 2015. We performed simple descriptive analysis on the data. We measured the assessment, report writing and follow-up against criteria for minimum standards for $\mathrm{CM}$ assessments, and identified whether assessments were child-friendly from available clinical information.

Results There were 304 children referred; 279 seen for acute assessment; most (73\%) were for sexual abuse, $75(27 \%)$ were for physical abuse/neglect. Over half the assessments identified other health concerns; joint assessments performed by paediatric and forensic doctors were better at identifying these health concerns than solo assessments. Most assessments were multidisciplinary and used protocols; half were not followed up; a third were performed after-hours and a third had no carer present during assessments.

Conclusions We identified strengths and weaknesses in current $\mathrm{CM}$ assessments in our service. Locally relevant standards for CM assessments are achievable in the acute setting, more challenging is addressing appropriate medical and psychosocial follow-up for these children. While we have established baseline domains for measuring a child-friendly approach to CM assessments, more should be done to ensure these vulnerable children are assessed in a timely, child-friendly manner, with appropriate follow-up.

\section{INTRODUCTION}

Since Henry Kempe drew global attention to physical abuse of children with the 'battered child syndrome', there has been an expansion in the literature within the field of child maltreatment $(\mathrm{CM}) \cdot{ }^{1-3}$ There is now clear

\section{What is already known on this topic?}

Children who have been maltreated have known health and developmental concerns.

- A comprehensive medical assessment is an essential component of a multi-agency child maltreatment (CM) assessment.

- There is wide variation in the quality of, and access to, CM clinical assessments.

\section{What this study hopes to add?}

Locally relevant standards for clinical assessment of $\mathrm{CM}$ in the acute setting can be achieved if clinicians are well supported.

- Joint assessments and multidisciplinary assessments are better at identifying other health concerns than solo assessments.

- Achieving appropriate medical and psychosocial follow-up of children following acute CM assessments is challenging.

- We have established simple baseline domains for the measurement of child-friendly CM assessments; more can be done to uphold children's rights in acute settings.

acknowledgement that $\mathrm{CM}$ is a global public health and social welfare problem, with known significant short-term, medium-term and long-term health consequences. ${ }^{4-8}$ As early as 1962, the role of medical professionals in their "duty and responsibility to the child to require a full evaluation of the problem' was identified. ${ }^{1}$ Over recent decades, there has been an increased understanding of the health needs of maltreated children, ${ }^{9} 10$ and expansion in clinical and forensic assessment guidelines. ${ }^{11-14}$

A comprehensive medical assessment is an acknowledged essential component of a multiagency investigation of CM. ${ }^{11}$ There is now good evidence for performing comprehensive medical assessments for acute presentations of CM, not just for forensic purposes but because of the high yield of health 
concerns identified. ${ }^{15-17}$ Despite the plethora of guidelines available, little is known about the quality dimensions of these assessments. While there have been many attempts made to improve the quality of detection of CM in emergency departments and front-line services, ${ }^{18} 19$ there has been little reported on improving the quality of medical assessments for acute CM presentations. Rose et al reported from their New Zealand study of child sexual assault (SA) wide regional variation in the proportion of children and adolescents receiving a medical assessment and variation in the quality of the service structure. ${ }^{16}$ Upholding children's rights is acknowledged as an essential component of paediatric quality of care, and some work has gone into assessing quality in paediatric hospital services with a focus on children's rights. ${ }^{20}$ Dimensions of children's rights include the assessment of equity of access, timeliness, effectiveness, safety, continuity of care, non-discrimination, child-friendly environment, communication, culturally appropriate and holistic care. Australian standards for the provision of child, adolescent and family-friendly health service facilities are available, ${ }^{21}$ but they tend to be focused on children's wards, play facilities and separation between children or adolescents and adults in hospitals. Little is known about how child-centred or children's rights promoting CM medical assessments are.

South Western Sydney (SWS) Local Health District is the largest, most populous health district in New South Wales (NSW), Australia, with a substantial child and youth population..$^{22}$ It is a rapidly growing metropolitan population in the state of NSW, with a large culturally and linguistically diverse population and many subgroups who are socially and economically at risk within its boundaries. ${ }^{23}$ Raman $e t a l^{24}$ previously identified and published locally relevant minimum standards for clinical assessment of child physical abuse and neglect (PAN) from a quality audit conducted in SWS. The authors highlighted wide variation in clinical practice in acute assessment and follow-up of children presenting at their most vulnerable to front-line clinical services. Following that audit, the Community Paediatrics team in SWS made improvements to the clinical assessment processes. A model of collaborative multidisciplinary clinical assessments for acute $\mathrm{CM}$ was established in one hospital (a non-tertiary paediatric hospital) setting; the partnership included community paediatricians (CPs), social workers, SA physicians and hospital-based paediatricians.

Our aims were to describe acute presentations of $\mathrm{CM}$ to this collaborative clinical service over a 3-year period. We wanted to determine whether the cases assessed fulfilled previously established minimum standards for clinical assessment of CM within our service to see whether service improvements had taken place. ${ }^{24}$ We also wanted to see whether the assessments were conducted in a child rights promoting or a childfriendly manner, within the constraints of an acute clinical setting. The results of this audit would further feed back into clinical service improvements in SWS, thereby contributing to and sustaining the quality improvement process. ${ }^{25}$

\section{METHODS}

As part of ongoing quality improvement initiatives in child protection, we audited acute presentations of CM to the unique collaborative clinical service in SWS between the years 2013 to 2015 . We collated data gathered from the acute child protection databases for all referrals for acute assessments to one hospital service in SWS of children and youth $(<16$ years) between 1 January 2013 to 31 December 2015, including PAN and SA. These data were derived from two databases; one established by CPs and the other from SA Services. PAN assessments were carried out by paediatricians, and SA assessments were carried out either by SA doctors (forensic physicians) alone, especially if they were urgent, or collaboratively by $\mathrm{CP}$ and SA doctors either due to the age of the child or due to complexity of the clinical presentation. Those children that appeared in both databases for the same assessment were categorised as a joint assessment conducted collaboratively by $\mathrm{CP}$ and SA physicians.

We reviewed all the clinical reports, clinical notes and follow-up plans of children seen for CM assessments. Demographics, referral details, forensic, clinical and social outcomes were recorded from the available data. We used the clinical findings of the medical or forensic notes and reports to classify the medical examination across several dimensions. The health needs identified in the reports were classified as medical, developmental/ learning and behavioural/mental health or a combination of two or all areas. The referring services were contacted by the researchers to find out what happened to the children following their acute assessments. The assessments were then reviewed to see whether certain minimum standards were achieved, taken from the previously established criteria (box) ${ }^{24}$ :

The medical records of the child, both hospital and SA records, were reviewed to see whether medical and psychosocial follow-up had occurred or not. If the follow-up had not occurred, the notes were reviewed to see whether an appointment had been offered, yet the

Box Minimum standards for the clinical assessment of child maltreatment

All children presenting with suspected significant child maltreatment (CM) or referred by Community Services are assessed by a paediatric trained doctor, social worker, \pm nurse as appropriate.

- All clinical assessments to follow clinical protocol.

- Assessments to be discussed with most senior consultant.

- If child protection report is to be generated, it needs to be countersigned by consultant.

- Protocols and report to be filed in the medical record.

- Paediatric and psychosocial follow-up to be available to all children identified with abuse and neglect, across the region. 
child was not brought to the appointment. The reports and $\mathrm{CM}$ assessments were reviewed to assess how childfriendly they were. We identified timeliness, appropriateness of assessment, presence of parent or carer, from relevant tools and standards which pertain to children's rights in healthcare. ${ }^{21}$ The measures used to determine how 'child-friendly' a CM assessment was included:

1. length of time to assessment

2. whether assessments were conducted within hours (Monday-Friday; 08:00 to 17:00) or after-hours

3. a carer or support person was present with the child during assessment.

Relevant data extracted from the databases were entered into an Excel spreadsheet, and simple descriptive analysis of the data was carried out using IBM SPSS Statistics for Windows V.23. $\chi^{2}$ tests and two-sample t-tests were used to determine difference in proportions and difference in means.

\section{RESULTS}

Over the 3-year period (2013-2015), 304 cases were referred for an acute CM assessment, of which $25(8 \%)$ were phone consultations. There were 279 cases seen for an acute assessment, 204 cases $(73 \%)$ were seen for suspected SA and 75 cases $(27 \%)$ were for suspected PAN assessments. Of the 279 cases, 15 cases (5\%) seen were siblings of an index child and one child was seen for neglect. A detailed description of the demographics, referral and assessment findings of the children has been presented before. ${ }^{26}{ }^{27}$ Median age of presentation for all types of assessments was 7 years (IQR of 3-13 years); median age of children presenting for all SA assessments was 10 years (IQR 4-14 years), for PAN was 3.5 years (Mann-Whitney $\mathrm{U}$ test $\mathrm{Z}$ score $=6.84, \mathrm{P}<0.0001$ ). The majority of the children seen for $\mathrm{CM}$ assessment were identified as Anglo-Australian ( $\mathrm{n}=77,27 \%)$, with high numbers of Aboriginal $(\mathrm{n}=39,14 \%)$ and Pacific Islander $(\mathrm{n}=35,13 \%)$ children. Ethnicity was not documented in $37 \%$ of sole SA assessments. Police and/or child protection agencies made $82 \%$ of the referrals for acute assessments. A third of the SA assessments were joint $(\mathrm{CP} / \mathrm{SA})$ assessments. A third of all assessments were for forensic purposes.
Other health concerns were identified in 151 (54\%) of the 279 children examined; learning difficulties were identified in 28 children (19\%); 21 children (14\%) had behavioural problems. Health concerns identified included growth and nutrition problems, dental caries, incomplete immunisation, pneumonia, hearing problems, visual problems, skin infections and current gastroenteritis. Physical health, learning difficulties and mental health concerns $(3+$ concerns) coexisted in 25 children $(17 \%)$. For SA assessments, joint assessments (86\%) identified more health concerns or were more likely to identify health concerns than those performed by sole SA doctors $(26 \%)\left(\chi^{2}=72.33, \mathrm{P}<0.001\right)$.

Table 1 shows the medical follow-up of unmet health concerns; the majority $(94 \%)$ of children with medical concerns were referred for medical follow-up with $70 \%$ of them being seen. All seven children with unknown follow-up were placed in out-of-home-care (OOHC). Also, 8 (22\%) of the 37 children that were not brought to follow-up were placed in OOHC, and 21 (57\%) of them went home with their family but with ongoing statutory child protection agency involvement.

Table 2 reports on various aspects of the clinical assessments judged against the predetermined criteria. Standards that were achieved for most assessments were that they were multidisciplinary, used protocol and had reports generated. SA reports that were not generated were due to child refusing medical examination and/or the case determined to be not consistent with abuse. All reports that were generated were countersigned by the senior consultant and placed into the medical records for that child. Both medical and psychosocial follow-up was less successful. Overall, 195/279 (70\%) children were offered a follow-up medical appointment, and of those, $120(62 \%)$ attended. Following the solo SA assessments, $82 / 134(61 \%)$ were offered and 45/82 (55\%) attended; of the joint SA assessments, $55 / 70(79 \%)$ were offered and 35/55 (64\%) attended; of PAN assessments, 58/75 $(77 \%)$ were offered and $40 / 58(69 \%)$ attended. Of the 53 children not offered a follow-up medical assessment after their CM assessment, 30 (57\%) had examinations that were not concerning or consistent with abuse and had no other unmet health needs identified. Two (11\%)

Table 1 Medical follow-up of identified health and developmental concerns

\begin{tabular}{lcclc}
\hline Health concerns & $\begin{array}{l}\text { Attended follow- } \\
\text { up assessment }\end{array}$ & $\begin{array}{l}\text { Offered follow- up, } \\
\text { was not brought* }\end{array}$ & $\begin{array}{l}\text { No follow-up } \\
\text { appointment offered up occurred }\end{array}$ \\
\hline Medical alone $n=40$ & $27(68 \%)$ & $9(23 \%)$ & 0 & $4(10 \%)$ \\
Learning difficulties alone $n=28$ & $19(68 \%)$ & $6(21 \%)$ & $1(3 \%)$ & $2(7 \%)$ \\
Behavioural problems alone $n=21$ & $17(81 \%)$ & $4(19 \%)$ & 0 & 0 \\
2 concerns $n=37$ & $26(70 \%)$ & $10(35 \%)$ & $1(3 \%)$ & 0 \\
3+ concerns $n=25$ & $16(64 \%)$ & $8(32 \%)$ & 0 & $1(4 \%)$ \\
Total heath concerns $n=151$ & $105(70 \%)$ & $37(24 \%)$ & $2(1 \%)$ & $7(5 \%)$ \\
\hline
\end{tabular}

`The term 'was not brought' preferred to 'did not attend' as these are dependent children. 
Table 2 Assessing whether minimum standards were achieved in child maltreatment clinical assessments

\begin{tabular}{|c|c|c|c|c|}
\hline Criteria & Sole SA $n=134$ & Joint SA $n=70$ & PAN $n=75$ & CM (total) $n=279$ \\
\hline Trained doctor completed assessment & $134(100 \%)$ & $70(100 \%)$ & $75(100 \%)$ & $279(100 \%)$ \\
\hline Social worker present & $134(100 \%)$ & $70(100 \%)$ & $62(83 \%)$ & $265(95 \%)$ \\
\hline Protocol used & $130(97 \%)$ & $70(100 \%)$ & $75(100 \%)$ & $275(99 \%)$ \\
\hline Case discussed with most senior doctor & $134(100 \%)$ & $70(100 \%)$ & $75(100 \%)$ & $279(100 \%)$ \\
\hline Report generated & $114^{*}(100 \%)$ & $70(100 \%)$ & $75(100 \%)$ & $259^{*}(100 \%)$ \\
\hline Attended medical follow-up assessment & $45(34 \%)$ & $35(50 \%)$ & $40(53 \%)$ & $120(43 \%)$ \\
\hline Offered follow-up but was not brought & $37(28 \%)$ & $20(29 \%)$ & $18(24 \%)$ & $75(27 \%)$ \\
\hline No follow-up appointment offered & $27(20 \%)$ & $14(20 \%)$ & $12(16 \%)$ & $53(19 \%)$ \\
\hline Unknown & $25(19 \%)$ & $1(1 \%)$ & $5(7 \%)$ & $31(11 \%)$ \\
\hline Attended psychological follow-up assessment & $73(55 \%)$ & $24(34 \%)$ & $4(5 \%)$ & $101(36 \%)$ \\
\hline Offered a follow-up but was not brought & $38(28 \%)$ & $14(20 \%)$ & $3(4 \%)$ & $55(19 \%)$ \\
\hline No follow-up appointment offered & $17(13 \%)$ & $24(34 \%)$ & $68(90 \%)$ & $109(39 \%)$ \\
\hline Unknown & $6(4 \%)$ & $8(12 \%)$ & $0(0 \%)$ & $14(5 \%)$ \\
\hline
\end{tabular}

${ }^{*}$ Only reports that were needed were generated.

$\mathrm{CM}$, child maltreatment; PAN, physical abuse and neglect; SA, sexual assault.

of the 53 children not offered medical follow-up did have unmet health needs identified. Overall 156 (56\%) of children seen acutely were offered psychological follow-up, of those $101(65 \%)$ attended. Following the solo SA assessments, 111/134 (82\%) were offered psychological follow-up and $73(66 \%)$ attended; following joint SA assessments, 38/70 (54\%) were offered psychological follow-up and $24(63 \%)$ attended. By contrast, 7 of the 75 (9\%) presenting for PAN assessments were offered psychosocial follow-up; there was a statistical difference compared with SA assessments $\left(\chi^{2}=90.3, \mathrm{P}<0.001\right)$.

Table 3 examines measurable aspects of the CM assessments that were child-friendly. The majority of CM assessments occurred within the recommended 24-72 hours; average was within 24 hours. After-hours assessments were more likely to be for SA $\left(\chi^{2}=14.71, \mathrm{P}<0.001\right)$, with half the SA assessments being conducted after-hours. This was mainly for forensic need, with 54 (78\%) of 69 afterhours SA assessments completed for a forensic purpose. Three of the eight (38\%) PAN assessments conducted after-hours were for forensic purposes. Over half of the children presenting for PAN assessments were unaccompanied by a carer or support person during the assessment; there was a significant difference compared with SA assessments $\left(\chi^{2}=19.76, \mathrm{P}<0.001\right)$.

\section{DISCUSSION}

We believe that this attempt to appraise the quality dimensions of CM medical assessments in a busy, metropolitan, non-tertiary paediatric service is unique. While there are established guidelines for peer review for paediatricians working clinically in child protection, ${ }^{28}$ improving the overall quality of multidisciplinary clinical assessments has not been reported. Our evaluation found that the acute CM assessments undertaken in our service fulfilled many of the previously identified locally relevant standards established for quality, but fell short in achieving our objectives of adequate and appropriate follow-up, both medical and psychological. Importantly, we attempted to establish some baseline criteria for assessing how childfriendly this clinical service was, using available audit data on timeliness and appropriateness of the assessment, and whether the child was supported by the presence of a parent or parent figure. Reading $e t a l^{29}$ argued strenuously for incorporating a children's rights view to widen the perspective on CM, thereby combining public-health and protective responses to CM. Certainly more can and should be done in this space.

We have already reported on the age, gender and ethnicity differentials in our clinic population. ${ }^{26} 27$

Table 3 Were acute child maltreatment clinical assessments child-friendly?

\begin{tabular}{lllll}
\hline & Sole SA $\mathbf{n = 1 3 4}$ & Joint SA $\mathbf{n = 7 0}$ & PAN $\mathbf{n = 7 5}$ & CM (total) $\mathbf{n = 2 7 9}$ \\
\hline Median time referral to assessment (days) & 0 & 1 & 0 & 0 \\
IQR in days & $0-0$ & $0-5$ & $0-1$ & $0-1$ \\
Assessments done after-hours & $67(50 \%)^{\star}$ & $2(3 \%)$ & $8(11 \%)$ & $77(28 \%)$ \\
Number with no carer/support person present & $19(14 \%)$ & $28(40 \%)$ & $38(51 \%)^{\star}$ & $85(30 \%)$ \\
\hline
\end{tabular}

${ }^{*} P$ value $<0.001$

$\mathrm{CM}$, child maltreatment; PAN, physical abuse and neglect; SA, sexual assault. 
Almost $40 \%$ of SA assessments undertaken by SA physicians did not have ethnicity documentation. We feel strongly that ethnicity and/or cultural identity documentation is an essential aspect of the quality of the paediatric health record. Over half the children assessed for $\mathrm{CM}$ had other health concerns identified, in keeping with other studies. ${ }^{1530}$ In our service, joint assessments (ie, those involving both paediatricians and forensic physicians) were more likely to identify other health concerns, suggesting that multidisciplinary collaborative assessments involving paediatricians, social workers and forensic physicians were better at identifying coexisting health concerns, as has been shown elsewhere. ${ }^{31}$ Forensic assessments for SA performed as solo examinations had a shorter mean time to assessment, suggesting that the main focus was on achieving the forensic medical examination in a timely manner. It is likely that there were more unmet health and developmental concerns in those children assessed for SA. The majority of children that had an unmet health concern identified had medical follow-up initiated as a result of the CM assessment which is similar to other studies. ${ }^{15} 17$ Findings from Thomas's study ${ }^{30}$ showed that over half of the unmet health needs identified at CM assessments were met in follow-up.

Minimum standards that we had previously identified for clinical CM assessments were mostly achieved in the acute setting; a pleasing result suggesting that many recommendations for CM assessments that had been put in place following the Raman et al study ${ }^{24}$ were implemented and working. Clinical assessments for CM in our service were found to be comprehensive, multidisciplinary, protocol driven and had senior consultant support. What remains a concern is the low number of both medical and psychosocial follow-up. One in five children seen for acute CM assessment was not offered medical follow-up and two in five were not offered follow-up psychological counselling. Of those that were offered follow-up, almost two-thirds attended medical and psychosocial follow-up. Follow-up of unmet health concerns was $70 \%$, and just under $60 \%$ of assessments that were not offered follow-up had no identified medical reason for follow-up. Follow-up remains an important part of $\mathrm{CM}$ assessments as there is a high risk of recurrence of maltreatment, ${ }^{32} 33$ and there are known long-term health and developmental consequences of CM. ${ }^{4734}$

The majority of the assessments occurred within the recommended 24-72hours. Joint assessments took longer to arrange; mostly due to the fact that these assessments were for historical sexual abuse. Over half the SA assessments were conducted after-hours; this was dictated by the need for a forensic examination including the collection of forensic samples. Getting forensic DNA may improve chances of substantiating the allegation of SA and lead to improvements in the overall criminal justice outcomes. Children are often brought to hospitals in the middle of the night unannounced by child protection statutory agencies and occasionally with no carer present. So health services have no initial control over when the child presents or who they present with. We identified that just under a quarter of SA assessments and $62 \%$ of PAN assessments that occurred after-hours could have been deferred to the following morning, based on whether there was acute forensic need. Clearly the service could improve in this regard and advocate for the child's physical and emotional needs during these difficult assessments. What is of greater concern is that nearly a third of all CM assessments and half of all PAN assessments had no accompanying carer or guardian present to provide emotional support to the child, or indeed to provide an adequate medical history for the child. This can add challenges to the service including obtaining consent and interpreting examination findings. While clinical staff would not be in a position to dictate who accompanies the child or in what manner the child is brought in, improvements in collaboration between health and welfare services are warranted to ensure that children are appropriately supported emotionally.

\section{LIMITATIONS}

This collaborative child protection clinical service in SWS is a unique service for CM assessments, and therefore, it may not reflect practice elsewhere. We had to rely on what was documented in the clinical records and documentation can be notoriously variable. ${ }^{35}$ Although follow-up in general was poor, it would seem that those with unmet health concerns identified may have been more proactively followed up. Children that had normal SA examinations with no other issues may have been referred and seen at another health service, such as sexual health centres. Likewise, some of the children that were placed in OOHC may have changed residential location and may have got their health needs addressed elsewhere. Nevertheless, the 15 children that were placed in OOHC who did not attend follow-up represent a failure of health and welfare systems collaborating in the best interests of the child. This is certainly an ameliorable issue if health and welfare services both acknowledged that medical and psychosocial follow-up were key performance indicators for their sectors and a measure of holistic care for vulnerable children.

\section{CONCLUSIONS}

The findings from this quality improvement project have already influenced clinical practice in child protection in this region. ${ }^{36}$ We have demonstrated that locally relevant standards for CM assessments in the acute setting are achievable; more challenging is addressing appropriate medical and psychosocial follow-up for these children. Our audit demonstrates that multidisciplinary or joint forensic/paediatric/social work examinations are better at identifying and responding to children's health needs, and therefore are in the best interests of the child. We know that children who have suffered abuse and neglect are more at risk of having their rights violated, ${ }^{37} 38$ and 
need special consideration in health settings. We have established some baseline domains for measurement for a child-friendly approach to CM assessments, and we can improve our own service delivery via better collaborative multidisciplinary action. However, further work is certainly warranted in improving the understanding of how the best interests of vulnerable children can be served in the acute assessment setting, particularly incorporating the views of children and young people themselves.

Contributors SR planned, conceived and designed the study, helped with data analysis and did the writing up. PRH helped with planning, did all the data gathering and analysis, helped with writing up.

Competing interests None declared.

Ethics approval Human Research Ethics Committee at Liverpool Hospital. Provenance and peer review Not commissioned; externally peer reviewed. Open Access This is an Open Access article distributed in accordance with the Creative Commons Attribution Non Commercial (CC BY-NC 4.0) license, which permits others to distribute, remix, adapt, build upon this work non-commercially, and license their derivative works on different terms, provided the original work is properly cited and the use is non-commercial. See: http://creativecommons.org/ licenses/by-nc/4.0/

(C) Article author(s) (or their employer(s) unless otherwise stated in the text of the article) 2017. All rights reserved. No commercial use is permitted unless otherwise expressly granted.

\section{REFERENCES}

1. Kempe $\mathrm{CH}$, Silverman FN, Steele BF, et al. The battered-child syndrome. JAMA 1962;181:17-24.

2. Skellern CY. Child protection: a 50-year perspective. J Paediatr Child Health 2015;51:87-90.

3. Scott DA. The landscape of child maltreatment. Lancet 2009;373:101-2.

4. Gilbert R, Widom CS, Browne K, et al. Burden and consequences of child maltreatment in high-income countries. Lancet 2009;373:68-81.

5. Cuijpers P, Smit F, Unger F, et al. The disease burden of childhood adversities in adults: a population-based study. Child Abuse Negl 2011:35:937-45.

6. Norman RE, Byambaa M, De R, et al. The long-term health consequences of child physical abuse, emotional abuse, and neglect: a systematic review and meta-analysis. PLoS Med 2012;9:e1001349.

7. Springer KW, Sheridan J, Kuo D, et al. Long-term physical and mental health consequences of childhood physical abuse: results from a large population-based sample of men and women. Child Abuse Negl 2007:31:517-30.

8. Fergusson DM, McLeod GF, Horwood LJ. Childhood sexual abuse and adult developmental outcomes: findings from a 30-year longitudinal study in New Zealand. Child Abuse Negl 2013;37:664-74.

9. Gilbert R, Kemp A, Thoburn J, et al. Recognising and responding to child maltreatment. Lancet 2009;373:167-80.

10. Jenny C. Committee on Child Abuse and Neglect, American Academy of Pediatrics. Recognizing and responding to medical neglect. Pediatrics 2007;120:1385-9.

11. RCPCH. Child protection companion. London: Royal College of Paediatrics and Child Health, 2006.

12. Adams JA, Kellogg ND, Farst KJ, et al. Updated guidelines for the medical assessment and care of children who may have been sexually abused. J Pediatr Adolesc Gynecol 2016;29:81-7.

13. Sittig JS, Post ED, Russel IM, et al. Evaluation of suspected child abuse at the ED; implementation of American Academy of Pediatrics guidelines in the Netherlands. Am J Emerg Med 2014;32:64-6.
14. Barker J, Hodes D. The child in mind: a child protection handbook. Revised edition ed. London: Routledge, 2004.

15. Kirk CB, Lucas-Herald A, Mok J. Child protection medical assessments: why do we do them? Arch Dis Child 2010;95:336-40.

16. Everitt R, Reed P, Kelly P. Medical assessment for child sexual abuse: a post-code lottery? J Paediatr Child Health 2012:48:389-94.

17. Al-Jilaihawi S, Borg K, Maguire S, et al. The value of paediatric assessment in historic child sexual abuse. Arch Dis Child 2017;102:550-5.

18. Benger JR. Quality improvement report: simple intervention to improve detection of child abuse in emergency departments. BMJ 2002;324:780-2.

19. Louwers EC, Korfage IJ, Affourtit MJ, et al. Accuracy of a screening instrument to identify potential child abuse in emergency departments. Child Abuse Negl 2014;38:1275-81.

20. Lazzerini M, Tamburlini G. Hospital care for children: quality assessment and improvement tool. Copenhagen: World Health Organization-Regional Office for Europe, 2015.

21. Hill MK, Pawsey M, Cutler A, et al. Consensus standards for the care of children and adolescents in Australian health services. Med J Aust 2011:194:78-82.

22. SWSLHD. Health profile of local communities. Sydney, Australia: South Western Sydney Local Health District, 2014.

23. Morgan K, Eastwood J, Faniran S. Headline population indicators data report 2009: a report for the South West Sydney region on key statistics relating to the health and wellbeing of its children and families. Liverpool, New South Wales, Australia: Karitane and the Department of Community Paediatrics, Sydney South West Area Health Service, 2010.

24. Raman S, Maiese M, Hurley K, et al. Addressing the clinical burden of child physical abuse and neglect in a large metropolitan region: improving the evidence-base. Soc Sci 2014;3:771-84.

25. NSW Health. Easy guide to clinical practice improvement: a guide for healthcare professionals. Sydney: Better Health Centre, NSW Health Department, 2002.

26. Hotton P, Raman S, Isaacs R, et al. Improving the clinical assessment of acute presentations of child maltreatment using a quality and child rights framework. ACCAN 2015: Cultural responsiveness in a multiagency world. Auckland, New Zealand: New Zealand Government, 2015.

27. Hotton PR, Raman S. Analysis of acute presentations for child protection medical assessments in a large, culturally diverse metropolitan setting. BMJ Paediatr Open 2017;1:e000120.

28. Thomas A, Mott A. Child protection peer review for paediatricians. Child Abuse Review 2013;22:60-6.

29. Reading R, Bissell S, Goldhagen J, et al. Promotion of children's rights and prevention of child maltreatment. Lancet 2009;373:332-43.

30. Thomas L. G164 Unmet health needs identified during child protection medical assessments and their follow-up: a one year retrospective review of medical reports. Arch Dis Child 2014:99(Suppl 1):A72-3.

31. Wallace GH, Makoroff KL, Malott HA, et al. Hospital-based multidisciplinary teams can prevent unnecessary child abuse reports and out-of-home placements. Child Abuse Negl 2007;31:623-9.

32. Hindley N, Ramchandani PG, Jones DP. Risk factors for recurrence of maltreatment: a systematic review. Arch Dis Child 2006;91:744-52.

33. White OG, Hindley N, Jones DP. Risk factors for child maltreatment recurrence: An updated systematic review. Med Sci Law 2015;55:259-77.

34. Etter DJ, Rickert VI. The complex etiology and lasting consequences of child maltreatment. J Adolesc Health 2013;53:S39-S41.

35. Lindberg DM, Lindsell CJ, Shapiro RA. Variability in expert assessments of child physical abuse likelihood. Pediatrics 2008;121:e945-e953.

36. Blackmore R. Change in child protection clinical practice in Liverpool Hospital. Liverpool, NSW: SWSLHD, 2016.

37. Waterston T, Yilmaz G. Child rights and health care. Child: Care, Health and Development 2014;40:1-3.

38. Raman $\mathrm{S}$, Woolfenden $\mathrm{S}$, Williams $\mathrm{K}$, et al. Human rights and child health. J Paediatr Child Health 2007:43:581-6. 